\title{
Changes in Resting Calcaneal Stance Position Angle Following Insole Fitting in Children With Flexible Flatfoot
}

\author{
Eui Chang Lee, MD, Myeong OK Kim, MD, PhD, Hyo Sang Kim, MD, Sang Eun Hong, MD \\ Department of Physical and Rehabilitation Medicine, Inha University School of Medicine, Incheon, Korea
}

\begin{abstract}
Objective To clarify the relationship of the initial radiologic and a biomechanical parameter at first clinical visit, and define the effectiveness of modified insole, following insole fitting in children with flexible flatfoot.

Methods Children aged less than 13 years with flexible flatfoot were enrolled. The total number of subjects was 66 (33 boys, 33 girls). The subjects were divided into 5 subgroups, based on age: 1-2, 3-4, 5-6, 7-9, and 10-12 years. The mean time period between the initial \& final examination for their resting calcaneal stance position angle (RCSPA) was 24 months. Radiography quantified the deformity by measuring angles, including the talometatarsal angle, the metatarsal angle, and the calcaneal pitch angle.

Results From the angles measured on radiographs, only the talometatarsal angle showed a statistically significant correlation to the initial RCSPA ( $\mathrm{r}=-0.578$ for right side, $\mathrm{r}=-0.524$ for left side; $\mathrm{p}<0.01$ ). The mean RCSPA improved in all subgroups of subjects following insole fitting. Moreover, in children younger than 7 years, the improvement in RCSPA from the insole fitting was greater compared to children aged 7 years and older.

Conclusion The insole has additionally beneficial effects in all populations younger than 13 years. However, there might exist a hidden effect of normal structural pedal alignment during growth accompanied with bony maturation and developmental process. To date, it is controversial whether the treatment of flexible flatfoot is necessary in the vast majority of cases, or simple observation and advice to parents would suffice.
\end{abstract}

Keywords Flatfoot, Insole, Parameters, Children

\section{INTRODUCTION}

Flatfoot results in problems on the static alignment of ankle and foot, as well as to the dynamic functional abnormality of the lower extremities [1]. Thus, proximal joints like the knee joints, hip joints, and the lower spine get affected due to the overloading of a medial longitudinal arch [2]. Proper diagnosis and subsequent manage-

Received May 23, 2016; Accepted August 31, 2016

Corresponding author: Myeong Ok Kim

Department of Physical and Rehabilitation Medicine, Inha University Hospital, 27 Inhang-ro, Jung-gu, Incheon 22332, Korea. Tel: +82-32-890-2480, Fax: +82-32-890-2486, E-mail: rmkmo@inha.ac.kr

ORCID: Eui Chang Lee (http://orcid.org/0000-0002-7383-0713); Myeong Ok Kim (http://orcid.org/0000-0002-5584-7714); Hyo Sang Kim (http://orcid. org/0000-0003-4071-6989); Sang Eun Hong (http://orcid.org/0000-0002-9433-6642).

(c) This is an open-access article distributed under the terms of the Creative Commons Attribution Non-Commercial License (http://creativecommons.org/ licenses/by-nc/4.0) which permits unrestricted noncommercial use, distribution, and reproduction in any medium, provided the original work is properly cited. Copyright (c) 2017 by Korean Academy of Rehabilitation Medicine 
ment for flexible flatfoot is therefore crucial for preventing these complications.

Despite a high prevalence of pediatric flatfoot, no general consensus exists on the diagnosis of flatfoot.

Clinically, flatfoot is diagnosed with the height of the medial longitudinal arch of the foot, evaluated by indirect or direct methods. An indirect method includes a footprint, and direct methods include biomechanical and radiographic parameters [3].

In a pediatric foot clinic, the resting calcaneal stance position angle (RCSPA) is a common biomechanical parameter to diagnose flatfoot. Sobel et al. [4] reported on the values of RCSPA of 212 people without flatfoot, aged from 5 to 36 years. The study concluded that variation among studies existed for the normal values of RCSPA, although generally the RCSPA has high degree of reliability, independent of age, height and weight.

Till date, there is no consensus on the radiological diagnosis of flatfoot. The calcaneal pitch angle (CPA), talometatarsal angle (TMA), and metatarsal angle (MA) are all considered to be helpful for diagnosing flatfoot in clinics [5]. Nevertheless, variability among researchers has been observed in the radiologic parameters used in clinics, and hence there is no universal agreement on normal values of radiologic parameters mentioned above, according to age.

Applying corrective insoles for children with flexible flatfoot still remains debatable among researchers. Moreover, the natural history of flexible flatfoot and correction of flatfoot by insole has been debated in many previous studies [2,6-15].

The objective of this study was to identify the changes of biomechanical parameters after applying modified insole in children with flexible flatfoot, and to assess the differences of these parameters following insole fitting, according to the age-matched subdivided groups.

\section{MATERIALS AND METHODS}

\section{Subjects}

Children who visited our foot clinic of Department of Physical and Rehabilitation Medicine, Inha University Hospital and were diagnosed with flexible flatfoot between January 2008 and December 2013, were recruited for this study. A total of 247 subjects were enrolled, aged 1 to 12 years. Patients diagnosed with other diseases (e.g., vertical talus, tarsal coalition, cerebral palsy, hypotonia, accessory navicular bone, etc.) were excluded from our study. Patients who had poor compliance of applying foot orthotics during the follow-up period were also excluded. Although none of the subjects complained of foot pain, some complained of fatigue when walking long distances. Overall, 66 children satisfied the RCSPA followup times of 24-57 months; these were the final number enrolled in the study. The final RCSPA was checked at 24 months for all subjects. The subjects were divided into age-matched 5 subgroups, based on the time of completion of normal longitudinal arch [6-9]: age groups 1-2, 3-4, 5-6 years (preschool age of which flexible flatfoot can be present in a normal situation), 7-9 years (the time when the maturation of medial longitudinal arch develops), and 10-12 years (the time when arch development is complete, and thus natural correction rarely occurs). Differences and changes of RCSPA were noted for all the age groups (Fig. 1). The subjects were also evaluated as 2 groups, based on the time of completion of normal longitudinal arch: preschool group from 1-6 years, and school age group from 7-12 years.

\section{Methods}

All subjects were examined by a single professional physician from the rehabilitation department. Flatfoot was diagnosed when the RCSPA was less than $0^{\circ}$ using the angle finder $[16,17]$.

RCSPA was serially measured at every clinical visit. The parameters used in the study were based on the initial and final values. The time for insole fitting was within the 1st month of the first clinical visit. In addition, a single

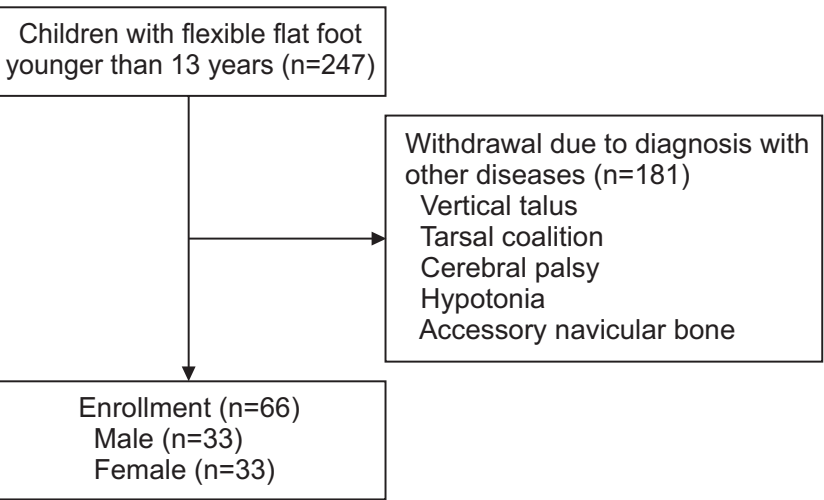

Fig. 1. Flow chart of study selection. 
professional physician measured all angles of radiography parameters to quantify the deformity, which included the TMA, MA and CPA.

\section{Measuring the RCSPA}

The RCSPA measurement was performed as follows.
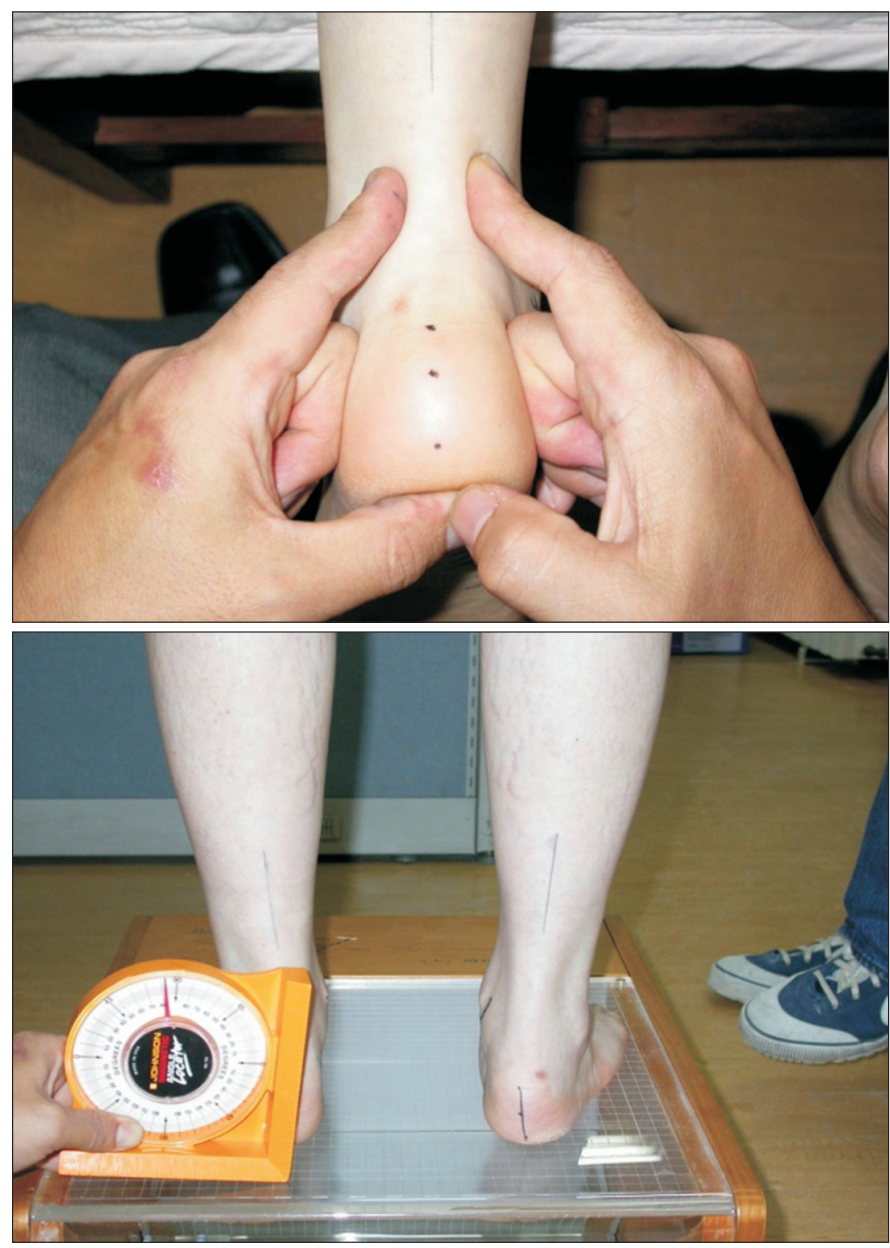

The patient is first relaxed and made to lie in the prone position on the examination table. Second, the physician plots three dots vertically on the foot: the upper, middle, and lower dots, bisecting the calcaneal bone using a bimanual technique; those dots are connected by drawing a line. Third, the patient is relaxed and positioned stand-

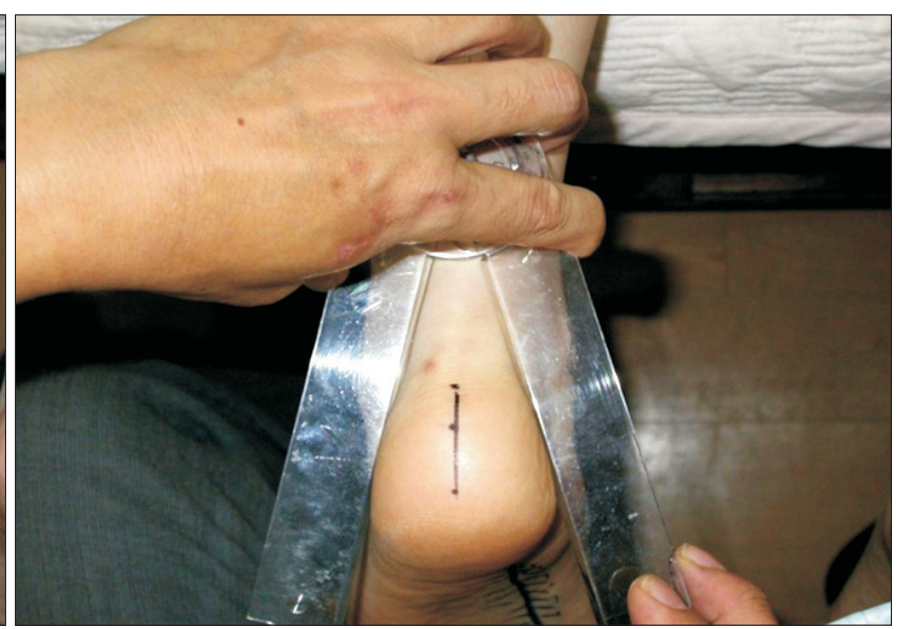

Fig. 2. Measurement of the resting calcaneal stance position angle (RCSPA).
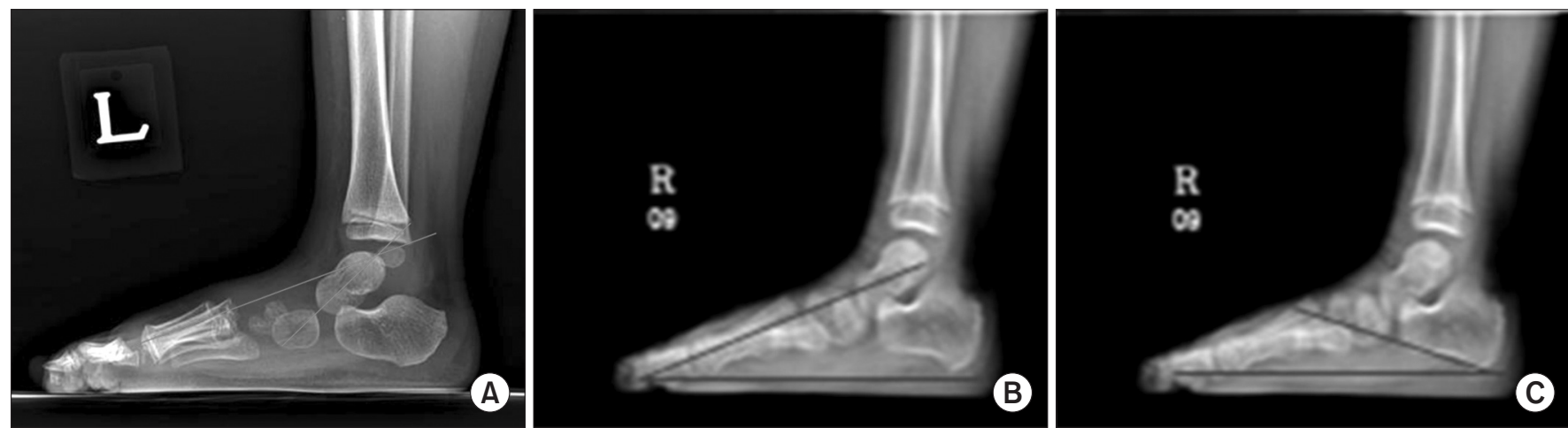

Fig. 3. (A) Talometatarsal angle, (B) metatarsal angle, and (C) calcaneal pitch angle. 
ing on his/her feet. Fourth, the physician measures the angle between the vertical and the ground and the calcaneal bisection line (Fig. 2).

\section{Radiological parameters}

Four lines were drawn in a standard weight-bearing lateral radiograph of the foot to demonstrate the medial longitudinal arch. The longitudinal axes of the first metatarsal bone, longitudinal axes of the talus, the horizontal baseline starting from the inferior process of the calcaneus which is parallel to the ground, and the calcaneal line, are drawn along the inferior surface of the calcaneus. The TMA is the angle between the line drawn from the centers of longitudinal axes of the talus and the first metatarsal bone. The MA is the angle between the longitudinal line of the first metatarsal bone and the horizontal baseline. The CPA is the acute angle between the inferior border of the calcaneus and the horizontal baseline (Fig. 3).

\section{Statistical analysis}

The Korean version of SPSS ver. 21.0 for Windows (IBM

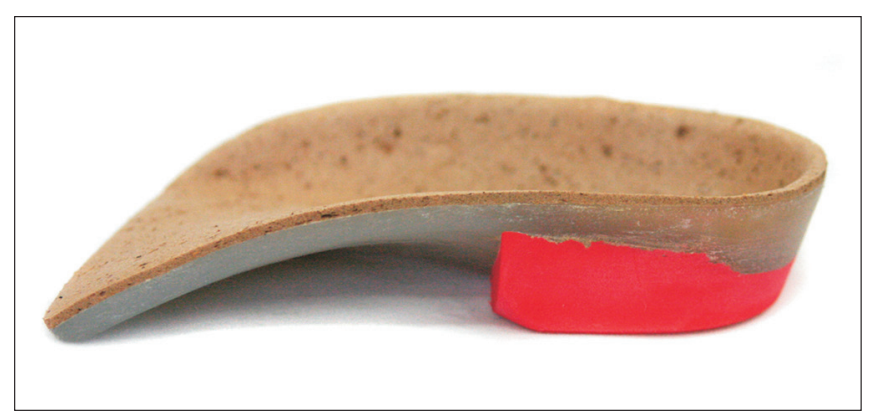

Fig. 4. Custom-made rigid foot orthosis manufactured on the basis of the inverted technique (Biomechanics, Goyang, Korea).
SPSS, Armonk, NY, USA) was used for statistical analysis. The correlation between the RCSPA measured at the first visit and the radiological parameters were analyzed using a Pearson correlation coefficient (r). We also analyzed the statistical significance of the difference between the RCSPA measured at the initial and final visits, using the paired t-test in all age groups. Furthermore, an independent $\mathrm{t}$-test was performed to show the statistical significance of the difference between the preschool group and the school age group. The statistical significance was defined when $\mathrm{p}<0.05$.

\section{Corrective insole}

When a biomechanical abnormality such as overpronation was found, a cast from a pair of custom-made rigid foot orthosis (RFO) was manufactured (Biomechanics, Goyang, Korea) on the basis of the inverted technique (Fig. 4), defined as the corrective insole. For example, when the initial RCSPAs were $-5^{\circ}$ to $-6^{\circ}$ and $-7^{\circ}$ to $-8^{\circ}$, the inverted orthotic technique prescribed $20^{\circ}$ and $25^{\circ}$ angles, respectively. For RCSPA of $-9^{\circ}$ to $-10^{\circ}$, and for the more severe type lower than $-11^{\circ}, 30^{\circ}$, and $35^{\circ}$ inverted orthotic technique with lateral long flanges were prescribed, respectively. Each subject was recommended to wear the orthoses for more than 8 hours per day, and to walk with a heel strike at initial contact and reciprocal arm swing, to normalize the gait pattern.

Every subject diagnosed with flexible flatfoot was provided with insoles at an average of 1 month after the first visit.

\section{RESULTS}

A total of 66 subjects were enrolled in the study, and

Table 1. Change of the RCSPA before and after insole fitting according to the age group

\begin{tabular}{lllll}
\hline \multirow{2}{*}{ Age group $(\mathbf{y r})$} & \multicolumn{4}{c}{ RCSPA $\left({ }^{\circ}\right)$} \\
\cline { 2 - 5 } & Initial right & Initial left & Final right & Final left \\
\hline $1-2(\mathrm{n}=13)$ & $-7.0 \pm 4.8$ & $-10.5 \pm 2.9$ & $-3.4 \pm 4.2^{*}$ & $-7.2 \pm 3.2^{*}$ \\
$3-4(\mathrm{n}=18)$ & $-7.1 \pm 3.6$ & $-10.1 \pm 3.7$ & $-3.4 \pm 2.5^{*}$ & $-5.9 \pm 3.0^{*}$ \\
$5-6(\mathrm{n}=18)$ & $-6.1 \pm 3.4$ & $-8.0 \pm 3.8$ & $-3.3 \pm 2.7^{*}$ & $-5.2 \pm 3.1^{*}$ \\
$7-9(\mathrm{n}=9)$ & $-5.3 \pm 2.0$ & $-8.0 \pm 2.8$ & $-3.2 \pm 1.9^{*}$ & $-6.1 \pm 1.7^{*}$ \\
$10-12(\mathrm{n}=8)$ & $-4.0 \pm 3.1$ & $-6.3 \pm 3.8$ & $-2.9 \pm 1.7^{*}$ & $-4.3 \pm 2.6^{*}$ \\
\hline
\end{tabular}

Values are presented as mean \pm standard deviation.

RCSPA, resting calcaneal stance position angle.

${ }^{*} \mathrm{p}<0.05$, initial right vs. final right, final right vs. final left. 
Table 2. Change of the RCSPA before and after insole fitting, according to the preschool group vs. the school age group

\begin{tabular}{|c|c|c|c|c|c|c|}
\hline & \multicolumn{4}{|c|}{$\operatorname{RCSPA}\left({ }^{\circ}\right)$} & \multicolumn{2}{|c|}{ Effectiveness } \\
\hline & Initial right & Initial left & Final right & Final left & Right & Left \\
\hline Group $1(n=49)$ & $-6.7 \pm 3.8^{\text {a) }}$ & $-9.4 \pm 3.6^{\text {a) }}$ & $-3.4 \pm 3.0^{\mathrm{b})}$ & $-6.0 \pm 3.1^{b)}$ & $-0.49 \pm 0.42^{c)}$ & $-0.38 \pm 0.24^{\mathrm{d})}$ \\
\hline Group $2(\mathrm{n}=17)$ & $-4.7 \pm 2.6^{\mathrm{a})}$ & $-7.2 \pm 3.3^{\mathrm{a})}$ & $-3.1 \pm 1.8^{\mathrm{b})}$ & $-5.2 \pm 2.3^{\mathrm{b})}$ & $-0.31 \pm 0.27^{\mathrm{c})}$ & $-0.26 \pm 0.12^{\mathrm{d})}$ \\
\hline
\end{tabular}

Values are presented as mean \pm standard deviation.

RCSPA, resting calcaneal stance position angle; group 1, age 1-6 years; group 2, age 7-12 years.

${ }^{a)} \mathrm{p}<0.05$, difference of initial right, initial left between group 1 and group 2.

${ }^{b)} \mathrm{p}>0.05$, difference of final right, final left between group 1 and group 2.

c) $\mathrm{p}<0.05$, difference of right effectiveness between group 1 and group 2 .

${ }^{d)} \mathrm{p}<0.05$, difference of left effectiveness between group 1 and group 2 .

Right effectiveness $=($ final RCSPA - initial RCSPA of right side $) /$ initial RCSPA of right side.

Left effectiveness $=($ final RCSPA - initial RCSPA of left side $) /$ initial RCSPA of left side.

Table 3. Initial radiological parameters before insole fitting according to the age group

\begin{tabular}{|c|c|c|c|c|c|c|}
\hline \multirow{2}{*}{ Age group (yr) } & \multicolumn{2}{|c|}{ TMA $\left(^{\circ}\right)$} & \multicolumn{2}{|c|}{ MA $\left(^{\circ}\right)$} & \multicolumn{2}{|c|}{ CPA $\left({ }^{\circ}\right)$} \\
\hline & Right & Left & Right & Left & Right & Left \\
\hline $1-2$ & $15.6 \pm 15.8$ & $12.3 \pm 9.8$ & $21.7 \pm 5.5$ & $23.9 \pm 4.2$ & $13.5 \pm 4.5$ & $18.1 \pm 6.9$ \\
\hline $3-4$ & $22.5 \pm 10.2$ & $20.2 \pm 11.7$ & $20.3 \pm 2.8$ & $20.9 \pm 3.4$ & $12.5 \pm 4.5$ & $16.7 \pm 5.6$ \\
\hline $5-6$ & $19.9 \pm 14.4$ & $16.5 \pm 11.6$ & $19.6 \pm 6.1$ & $20.9 \pm 4.0$ & $11.3 \pm 7.7$ & $12.3 \pm 4.1$ \\
\hline $7-9$ & $6.6 \pm 7.0$ & $6.6 \pm 4.0$ & $23.4 \pm 4.7$ & $21.5 \pm 5.9$ & $18.3 \pm 8.7$ & $18.9 \pm 6.7$ \\
\hline $10-12$ & $6.9 \pm 2.8$ & $7.0 \pm 2.1$ & $20.6 \pm 2.7$ & $18.8 \pm 0.6$ & $15.2 \pm 3.2$ & $13.4 \pm 1.3$ \\
\hline
\end{tabular}

Values are presented as mean \pm standard deviation.

TMA, talometatarsal angle; MA, metatarsal angle; CPA, calcaneal pitch angle.

Table 4. Correlation coefficient (r) between initial RCSPA score and radiologic parameters

\begin{tabular}{|c|c|c|c|c|c|c|}
\hline \multirow{2}{*}{ RCSPA } & \multicolumn{2}{|c|}{ TMA } & \multicolumn{2}{|c|}{ MA } & \multicolumn{2}{|c|}{ CPA } \\
\hline & Right & Left & Right & Left & Right & Left \\
\hline Initial right & $-0.578^{*}$ & $-0.524^{*}$ & 0.265 & 0.124 & -0.111 & 0.040 \\
\hline Initial left & $-0.349^{*}$ & $-0.433^{*}$ & 0.116 & 0.034 & -0.072 & 0.002 \\
\hline
\end{tabular}

RCSPA, resting calcaneal stance position angle; TMA, talometatarsal angle; MA, metatarsal angle; CPA, calcaneal pitch angle.

${ }^{*} \mathrm{p}<0.05$.

classified into groups as per their age: 1-2 years (13 subjects), 3-4 years (18), 5-6 years (18), 7-9 years (9), and 10-12 years (8). Mean age was 5.0 \pm 2.7 years. Of the 66 subjects, 60 had a dominant foot on the right side.

\section{RCSPA}

The follow-up time of the RCSPA was 24 months. The average RCSPA at the initial visit was $-6.2^{\circ} \pm 3.6^{\circ}$ on the right, and $-8.9^{\circ} \pm 3.7^{\circ}$ on the left, and at the final visit average RCSPA was $-3.2^{\circ} \pm 2.7^{\circ}$ on the right, and $-5.7^{\circ} \pm 2.9^{\circ}$ on the left. The RCSPA before and after insole fitting accord- ing to the 5 age-matched groups, are shown in Table 1 . The RCSPA differences between initial and final visit were statistically significant in all the 5 groups $(\mathrm{p}<0.05)$. Table 2 shows the RCSPA before and after insole fitting, according to the preschool age group versus school age group. The initial RCSPA differences between the preschool age group and school age group were statistically significant $(p<0.05)$. However, the final RCSPA differences between the two groups were not statistically significant $(p>0.05)$. The RCSPA difference, which is (Final RCSPA - Initial RCSPA) / Initial RCSPA, was greater in the preschool 
group than in the school age group, the difference being statistically significant $(\mathrm{p}<0.05)$ (Table 2$)$. In addition, the measurements at the final visit significantly improved, as compared with the initial measurement in all age groups.

\section{The relationship between RCSPA and radiological} parameters

Radiological parameters before insole fitting are shown in Table 3. The RCSPA measured at the first visit and the radiological parameters were analyzed using a Pearson correlation coefficient (r) (Table 4). The correlation coefficient of RCSPA and the three parameters observed are as follows: RCSPA and TMA (-0.58 in right, -0.43 in left); RCSPA and MA (0.26 in right, 0.03 in left); and RCSPA and CPA (-0.11 in right, 0.02 in left). According to the analysis, the TMA was statistically most significant among the three parameters on lateral radiographs.

\section{DISCUSSION}

Despite an absence of the definition of flatfoot, flexible flatfoot is reported in most children under the age of 10 years, and in at least $20 \%$ of the adult population $[18,19]$. However, clinical or radiological diagnosis on flatfoot has not reached any agreement within the research world. Therefore, the exact prevalence of the disorder is unknown. An epidemiological study revealed that flatfoot is thought to persist in $3 \%$ of the adult population [6].

Although radiographic investigations are not necessary for diagnosis, they can help in the assessment of decreased flexibility, uncharacterized pain, and surgical planning [20]. Since no consensus on the diagnosis of flatfoot exists, radiographic evaluations are often used in foot clinics as a reference to determine the magnitude of flatfoot. In a previous study, Kim et al. [21] evaluated the weight-bearing lateral views of the foot in 52 patients with flatfoot, and compared them to 60 control groups; they concluded that the TMA and MA could be used in clinical practice, as radiologic parameters of diagnosis in all age populations.

This study was conceived and undertaken since researches on the correlation of RCSPA, which is a common method for diagnosis of flatfoot in clinics and includes radiologic parameters, are very few. In our study, among the three lateral views of radiologic parameters, the TMA exhibited a significant correlation with RCSPA $(p<0.05)$.
Although flatfoot is often asymptomatic, foot pain is a frequent complaint during clinical assessment, especially after intense workouts or walking for a long time.

Flatfoot has been associated with problems in the ankle and foot structures, and further overloading could be transferred to more proximal lower extremities [22]. Researchers have found that patients who presented with a moderate to severe flatfoot tended to have anterior knee joint pain and intermittent low back pain, twice as much as controls $[7,23]$. However, it is not yet possible to predict the exact risks of disability or pain evoked by common foot disorders like flatfoot. When associated with severe foot pain, flatfoot can be referred for surgical intervention, but this is rare [6]. Although the diagnostic criteria vary among researchers, it is anticipated that the majority of flexible flatfeet are physiologic and resolve spontaneously with age, without further treatment.

Most flexible flatfeet are physiologic and asymptomatic, and thus require no treatment. However, clinically, the patients should be closely monitored for any possible symptoms or signs of progression [8,9,24]. Children with symptomatic flexible flatfoot have a long imposed controversy-what is pathologic, when to treat or observe, how to treat, and when to undergo surgery [24].

Applying foot orthotics for children with flexible flatfoot is still debatable. Many researchers believe that flatfoot resolves spontaneously over time [25]. Mosca et al. [6] found that foot orthotics helped in correcting the soft tissue deformity, but there was no change in the skeletal structure. Kwon and Myerson [26] reported that radiologic parameters did not improve in patients applying over-the-counter insole and foot orthotics, as compared to patients without any management. In general, it is believed that foot orthoses are not required in patients with asymptomatic flatfoot. Evidence also indicates that early application of orthotics prevents the development of symptomatic flatfoot [27].

Conversely, some previous studies have provided scientific evidence of prescribing foot orthoses. Halabchi et al. [5] proposed an algorithm for treating flexible flatfoot in children afflicted aged over 8 years, that needs to be treated despite lack of symptoms. Also, once imaging studies exclude any underlying structural abnormality which requires surgical intervention, active management like orthoses should be applied to patients with any symptoms related to flatfoot. Tang et al. [10] reported that excessive 
valgus movement of the rearfoot decreased significantly after applying forefoot medial posting orthoses in 10 patients with symptomatic flexible flatfoot. Aboutorabi et al. [11] enrolled 30 children with flatfoot, and divided them into groups with foot orthoses, regular shoe, and barefoot, to show the effectiveness of foot orthoses. Various gait parameters were used, such as step length and width, walking velocity, symmetry, and center of pressure $(\mathrm{CoP})$ displacement, to observe the difference among subjects. The group applying functional foot orthoses realized improvement in all parameters, as compared to the control group. In a recent study, Banwell et al. [12] asserted that not much evidence was found in the use of orthoses for flexible pes planus. However, some evidence indicated an improvement of physical function and energy cost during walking. In a previous study, substantial improvement was realized after applying customized foot orthoses to correct the RCSPA, with immediate improvement of the RCSPA [13-15]. Through the use of a dynamic stabilizing insole and customized foot orthoses to correct the RCSPA to the vertical position for flexible flatfoot patients, objective evidence in the improvement of foot prints and RCSPA was found [20].

In this study, the RCSPA values of both the right and left decreased in all age groups after adjusting insoles. As shown in Table 1, a more severe degree (in terms of the RCSPA) was found in the left foot compared to the right foot in all age groups of flatfoot children. The correction rate of the RCSPA was more significant in the left foot compared to the right foot. The findings of this study corresponded with a previous study [16]. Zifchock et al. [28] found that the height of the longitudinal arch was higher in dominant foot (mostly right foot) than in the nondominant foot (mostly left foot). The dominant foot is believed to be suitable for more delicate movement. On the other hand, the non-dominant foot is more appropriate for weight-bearing function. Overloading of a medial longitudinal arch makes the foot more pronated, which can explain why the RCSPA of left feet is smaller than that of right feet in most of subjects with flexible flatfoot in the study. In this study, 60 subjects among the 66 enrolled, had a dominant foot on the right side, which correlated with the results mentioned above.

A decrease in the RCSPA and the TMA was relatively large as age increased to 7 years. Contrastingly, a drop in the RCSPA was lesser in children older than 7 years, com- pared to that of children younger than 7 years. The results correlated with the previous studies that the normal developmental process of longitudinal arch begins around the age of 6 , and is completed before the age of 10 [6$9,19,28]$. However, this correction of the RCSPA and the TMA is still debatable, whether it is because of a natural history of flatfoot or because of the effectiveness of a corrective insole.

We divided our subjects by 5 age-matched groups in the study. We also assessed by dividing into 2 groups, based on the time of completion of normal longitudinal arch: preschool group from 1-6 years, and school age group from 7-12 years (Table 2). This was done to differentiate the effectiveness of insoles in 1-6 years from the combined effects of normal developmental process and insole effect in 7-12 years. As seen in Table 2, The RCSPA difference [(Final RCSPA - Initial RCSPA) / Initial RCSPA] was greater in the preschool group than that of the school age group. Although natural developmental process cannot be ruled out completely, comparing the RCSPA difference among subgroups according to the age, corrective insoles were considered to have additional effect for children with flexible flatfoot. According to Mosca [6], after the age of 6, RCSPA is supposed to normalize until age of around 10 years. After the age of 10 years, RCPSA remains unchanged. Therefore, the group aged from 10-12 years is thought to be able to exclude normal physiological maturation of the arch [6-9,19,28].

Our study has some limitations. First, although the RCSPA was found to be a reliable measurement when used by the same examiner (intra-tester reliability) [4], some errors are found in the interpretation of the results and the process of drawing conclusions, since this study was conducted in a situation where no consensus exists. Second, a control group was not involved in the study, as this research was conducted retrospectively. Thus, further study involving a control group is necessary to make conclusions regarding the effectiveness of insoles. Third, since this study focused on the changes of RCSPA following insole fitting in children with flexible flatfoot, other factors that might possibly affect changes of RCSPA, such as duration of follow-up, severity of flexible flatfoot and associated ligament laxity, were overlooked. Fourth, radiographic evaluations were planned for the final visit to demonstrate the changes of radiologic parameters following insole fitting in children with flatfoot. However, 
the concern of parents regarding radiation hazard and follow-up loss, made a repetitive evaluation difficult. Fifth, this research is a retrospective study, thereby lacking a number of subjects, especially in age group between 7-9 years and between 10-12 years. Thus, an accurate comparison was difficult among all age groups. In addition, the follow-up period for the RCSPA and the lateral view of radiographs varied among the subjects. Consequently, more prospective studies are required in future, with larger samples, longer follow-ups and the use of control groups, to confirm the therapeutic effects of an insole for flexible flatfoot.

In conclusion, this paper demonstrated that corrective insoles had an additional beneficial effect for flexible flatfoot in children younger than 13 years. However, there might be a hidden effect of normal structural pedal alignment during growth, accompanied with bony maturation and developmental process. Controversy still exists whether the treatment of flexible flatfoot is necessary in the vast majority of cases, or simple observation and advice to parents is sufficient. Further research needs to employ a prospective study which includes control groups, to discover the effectiveness of insoles and correlate the changes with biomechanical parameters.

\section{CONFLICT OF INTEREST}

No potential conflict of interest relevant to this article was reported.

\section{REFERENCES}

1. Lee JH, Sung IY, Yoo JY. Clinical or radiologic measurements and 3-D gait analysis in children with pes planus. Pediatr Int 2009;51:201-5.

2. Chen YC, Lou SZ, Huang CY, Su FC. Effects of foot orthoses on gait patterns of flat feet patients. Clin Biomech (Bristol, Avon) 2010;25:265-70.

3. Kanatli U, Yetkin H, Cila E. Footprint and radiographic analysis of the feet. J Pediatr Orthop 2001;21:225-8.

4. Sobel E, Levitz SJ, Caselli MA, Tran M, Lepore F, Lilja $\mathrm{E}$, et al. Reevaluation of the relaxed calcaneal stance position: reliability and normal values in children and adults. J Am Podiatr Med Assoc 1999;89:258-64.

5. Halabchi F, Mazaheri R, Mirshahi M, Abbasian L. Pediatric flexible flatfoot: clinical aspects and algorith- mic approach. Iran J Pediatr 2013;23:247-60.

6. Mosca VS. Flexible flatfoot in children and adolescents. J Child Orthop 2010;4:107-21.

7. Harris EJ, Vanore JV, Thomas JL, Kravitz SR, Mendelson SA, Mendicino RW, et al. Diagnosis and treatment of pediatric flatfoot. J Foot Ankle Surg 2004;43:341-73.

8. Cappello T, Song KM. Determining treatment of flatfeet in children. Curr Opin Pediatr 1998;10:77-81.

9. Sullivan JA. Pediatric flatfoot: evaluation and management. J Am Acad Orthop Surg 1999;7:44-53.

10. Tang SF, Chen CH, Wu CK, Hong WH, Chen KJ, Chen CK. The effects of total contact insole with forefoot medial posting on rearfoot movement and foot pressure distributions in patients with flexible flatfoot. Clin Neurol Neurosurg 2015;129 Suppl 1:S8-11.

11. Aboutorabi A, Saeedi H, Kamali M, Farahmand B, Eshraghi A, Dolagh RS. Immediate effect of orthopedic shoe and functional foot orthosis on center of pressure displacement and gait parameters in juvenile flexible flat foot. Prosthet Orthot Int 2014;38:218-23.

12. Banwell HA, Mackintosh S, Thewlis D. Foot orthoses for adults with flexible pes planus: a systematic review. J Foot Ankle Res 2014;7:23.

13. Jay RM, Schoenhaus HD, Seymour C, Gamble S. The Dynamic Stabilizing Innersole System (DSIS): the management of hyperpronation in children. J Foot Ankle Surg 1995;34:124-31.

14. Mereday C, Dolan CM, Lusskin R. Evaluation of the University of California Biomechanics Laboratory shoe insert in "flexible" pes planus. Clin Orthop Relat Res 1972;82:45-58.

15. Selby-Silverstein L, Hillstrom HJ, Palisano RJ. The effect of foot orthoses on standing foot posture and gait of young children with Down syndrome. NeuroRehabilitation 2001;16:183-93.

16. Shin JB, Kim SW, You S, Lee SK, Kim HS. Biomechanic analysis of lower extremities in children and teenagers with pes planus. J Korean Acad Rehabil Med 2008;32:154-9.

17. Ko YJ, Kim HW. Physical examination of foot and ankle. J Korean Assoc Pain Med 2006;5:1-7.

18. Gould N, Moreland M, Alvarez R, Trevino S, Fenwick J. Development of the child's arch. Foot Ankle 1989;9:241-5.

19. Staheli LT, Chew DE, Corbett M. The longitudinal arch: a survey of eight hundred and eighty-two feet 
in normal children and adults. J Bone Joint Surg Am 1987;69:426-8.

20. Evans AM, Rome K. A Cochrane review of the evidence for non-surgical interventions for flexible pediatric flat feet. Eur J Phys Rehabil Med 2011;47:69-89.

21. Kim SB, Yoon K, Park HS, Kwak H, Ha NJ, Park JS. Radiologic measurement of flatfoot. J Korean Acad Rehabil Med 2000;24:995-1001.

22. Fabry G. Clinical practice: static, axial, and rotational deformities of the lower extremities in children. Eur J Pediatr 2010;169:529-34.

23. Yagerman SE, Cross MB, Positano R, Doyle SM. Evaluation and treatment of symptomatic pes planus. Curr Opin Pediatr 2011;23:60-7.
24. Labovitz JM. The algorithmic approach to pediatric flexible pes planovalgus. Clin Podiatr Med Surg 2006;23:57-76.

25. Pfeiffer M, Kotz R, Ledl T, Hauser G, Sluga M. Prevalence of flat foot in preschool-aged children. Pediatrics 2006;118:634-9.

26. Kwon JY, Myerson MS. Management of the flexible flat foot in the child: a focus on the use of osteotomies for correction. Foot Ankle Clin 2010;15:309-22.

27. Mortazavi SJ, Espandar R, Baghdadi T. Flatfoot in children: how to approach? Iran J Pediatr 2007;17:163-70.

28. Zifchock RA, Davis I, Hillstrom H, Song J. The effect of gender, age, and lateral dominance on arch height and arch stiffness. Foot Ankle Int 2006;27:367-72. 